\title{
Las partículas modales del alemán: una revisión crítica
}

\author{
José Torregrosa Azor (Barcelona)
}

\begin{abstract}
One of the main features on which there seems to be a great agreement among researchers is that German modal particles are mostly used in oral language. However, after reviewing the literature ever since one of the most representative works in this field was made and after considering the characteristics that are derived and that define this group of words as a distinct group from other grammatical categories, we observe, on the one hand, that the relationship between German modal particles and intonation is an aspect hardly developed and, on the other hand, that this relationship is analysed taking into account a corpus of written data.

In this paper we purpose to change this point of view and recover the initial assumption. German modal particles would have to be analysed taking into account their natural context where they are used and, in order to do that, they would have to be studied exploring the interrelations between several oral codes, because it is there where their different modal meanings arise.
\end{abstract}

\section{$1 \quad$ Estado de la cuestión}

Las partículas del alemán es un campo de estudio complejo que ha sido tratado desde diferentes enfoques y métodos lingüísticos. A lo largo de la historia y especialmente durante los últimos cincuenta años ha suscitado no poca controversia por lo que respecta a los numerosos intentos de llevar a cabo su descripción y clasificación lingüística. A pesar de la cantidad ingente de trabajos que se han derivado relacionados con este tema, continúan existiendo interrogantes sobre la didáctica de estos elementos en un contexto enseñanza/aprendizaje del alemán como lengua extranjera y otros concernientes a su traducción e interpretación en otras lenguas. Sin embargo, además de todas estas cuestiones abiertas, habría que incluir otra de carácter metodológico que intentaremos fundamentar, comentar y, finalmente, proponer a lo largo del presente artículo.

A finales de la década de los sesenta del siglo veinte se experimenta un cambio de enfoque teórico y metodológico; el denominado kommunikative-pragmatische Wende (=cast. 'cambio comunicativo-pragmático') ${ }^{1}$ (cf. Helbig 1994: 15-16). Este cambio de orientación viene determinado por el interés centrado en estudiar la forma oral de la lengua y las diferentes funciones que desempeña según el contexto comunicativo en que se aplica, dando lugar a la creación de un marco teórico nuevo que promueve el desarrollo de la lingüística pragmática. El foco de atención de las investigaciones lingüísticas se centra en el análisis de la lengua oral.

\footnotetext{
${ }^{1}$ En adelante, junto al término utilizado en alemán se indicará la traducción al castellano entre paréntesis, tal y como se indica en el texto principal. La traducción al castellano de expresiones, frases u oraciones en alemán se indicará en nota al pie de página para facilitar la lectura y no recargar en exceso el texto principal.
}

Linguistik online 65, 3/14 - http://dx.doi.org/10.13092/lo.65.1404

licensed under CC 3.0 
Es justamente a partir de este momento, además de otras contribuciones significativas que se producen con anterioridad - principalmente de lingüistas soviéticos y las tesis de Kriwonossow (1977a) y de Weydt (1969) -, en que la investigación de las partículas del alemán adquiere un interés especial por diferentes motivos.

En primer lugar, las partículas del alemán no habían pasado inadvertidas por investigadores adscritos a la normativa estilística, cuyas contribuciones promovían prescindir de su uso. De forma particular, las partículas modales del alemán (en adelante PM) son consideradas palabras absolutamente carentes de sentido que no desempeñan ningún tipo de función, sino la de actuar como palabras de relleno; en consecuencia, el uso de las PM corresponde a la manifestación propia de un estilo descuidado e impropio que conviene evitar.

De esta forma, esta perspectiva ha dado lugar a diferentes designaciones terminológicas cuyo denominador común se caracteriza por una apreciación peyorativa de este grupo de palabras. Así, se pueden encontrar designaciones tales como Flickwörter (=cast. 'palabras de remiendo o partículas expletivas') (cf. Von der Gabelentz 1969; Reiners 1961: 339); farblose Redefüllsel (=cast. 'rellenos discursivos sin color') (cf. Lindqvist 1961: 24); Würzwörter (=cast. 'palabras condimento') (cf. Thiel 1962); Füllwörter (=cast. 'palabras de relleno o partículas expletivas') (cf. Adler 1964); Färbewörter (=cast. 'palabras colorido') (cf. Colditz 1966); o expresiones tales como que este tipo de palabras "pululan como piojos por la piel de nuestra lengua" 2 o que las PM representan "un fastidio"3 para los representantes de la estilística normativa que incluso recomiendan renunciar a su uso, sobre todo en el ámbito de la expresión escrita.

En segundo lugar, las investigaciones de Kriwonossow (1977a) y de Weydt (1969) se desmarcan de esta perspectiva excluyente y sellan el inicio de una nueva etapa, que suscita un interés inusitado por la investigación de las PM y por las partículas en general. El cambio de orientación teórica y metodológica que se produce a finales de los años sesenta del siglo XX posibilita centrar el interés precisamente en estos elementos de la lengua oral cuya importancia radica en la funcionalidad comunicativa y el significado pragmático que se vehicula a través de su emisión en un determinado contexto comunicativo.

Esta nueva situación propició la realización de una gran cantidad de trabajos sobre el tema en los que se adoptan diferentes perspectivas y métodos, cosa que ha dado lugar a proponer incluso que el estudio de las partículas debiera ser tratado como un ámbito de investigación específico y diferenciado (cf. Franck 1979: 11-13 y Weydt 1981: 47, Partikelforschung (=cast. 'investigación sobre partículas') Weydt 1981: 46 Partikologie (=cast. 'Particulogía'4)).

Sin embargo, las diferentes contribuciones que se han llevado a cabo para describir, caracterizar, clasificar y definir el grupo de las partículas y de las PM en particular como un grupo de palabras diferenciado del resto adoptaron criterios muy diversos que, a su vez, han derivado en la aplicación de diferentes enfoques metodológicos y también en la propuesta de una amplia variedad terminológica, aparte de la ya existente, que dificulta notablemente su estudio.

El panorama que se le ofrece al investigador interesado en el estudio de las partículas o de las PM es extremadamente complejo, ya que según el enfoque que se adopta para realizar su descripción lingüística se derivan propuestas muy diversas que complican la investigación. Desde el punto de vista de un no-germanohablante la sensación que se percibe es de tal

\footnotetext{
2 Traducción del original en alemán: "All diese Flickwörter wimmeln wie Läuse in dem Pelz unserer Sprache herum." (cf. Reiners 1961: 340).

3 Traducción del original en alemán: "sie [las PM] ein Dorn im Auge waren" (cf. Beerbom 1991: 25).

${ }^{4}$ No existe en castellano un término equivalente al concepto alemán. La solución que se ofrece aquí pretende reflejar el sentido que se vehicula mediante la propuesta alemana.
} 
dispersión y heterogeneidad que parece imposible determinar unos parámetros clasificatorios y establecer claramente unos límites adecuados para este grupo de palabras.

Además, los criterios gramaticales que se establecen para la clasificación del grupo Partikeln (=cast. 'partículas') no parecen ser universales en comparación con otras lenguas, sino que lo que, por ejemplo, en castellano se entiende por partícula, se conceptualiza en general de forma diferente en el ámbito lingüístico germano. Así, mientras que en castellano o en otras lenguas románicas los morfemas, los prefijos y sufijos son considerados partículas, este no es el caso de la lengua alemana, que las considera de forma generalizada como palabras completas o lexemas en un sentido amplio del término. Sin embargo, Erben (1958) sí considera los prefijos verbales dentro del grupo de partículas, según observa Kriwonossow (1977a: 9). Como consecuencia, el inventario que se propone de este grupo de palabras es muy diverso y los inventarios que son coincidentes se debe a que los autores adoptan un mismo enfoque metodológico para su análisis.

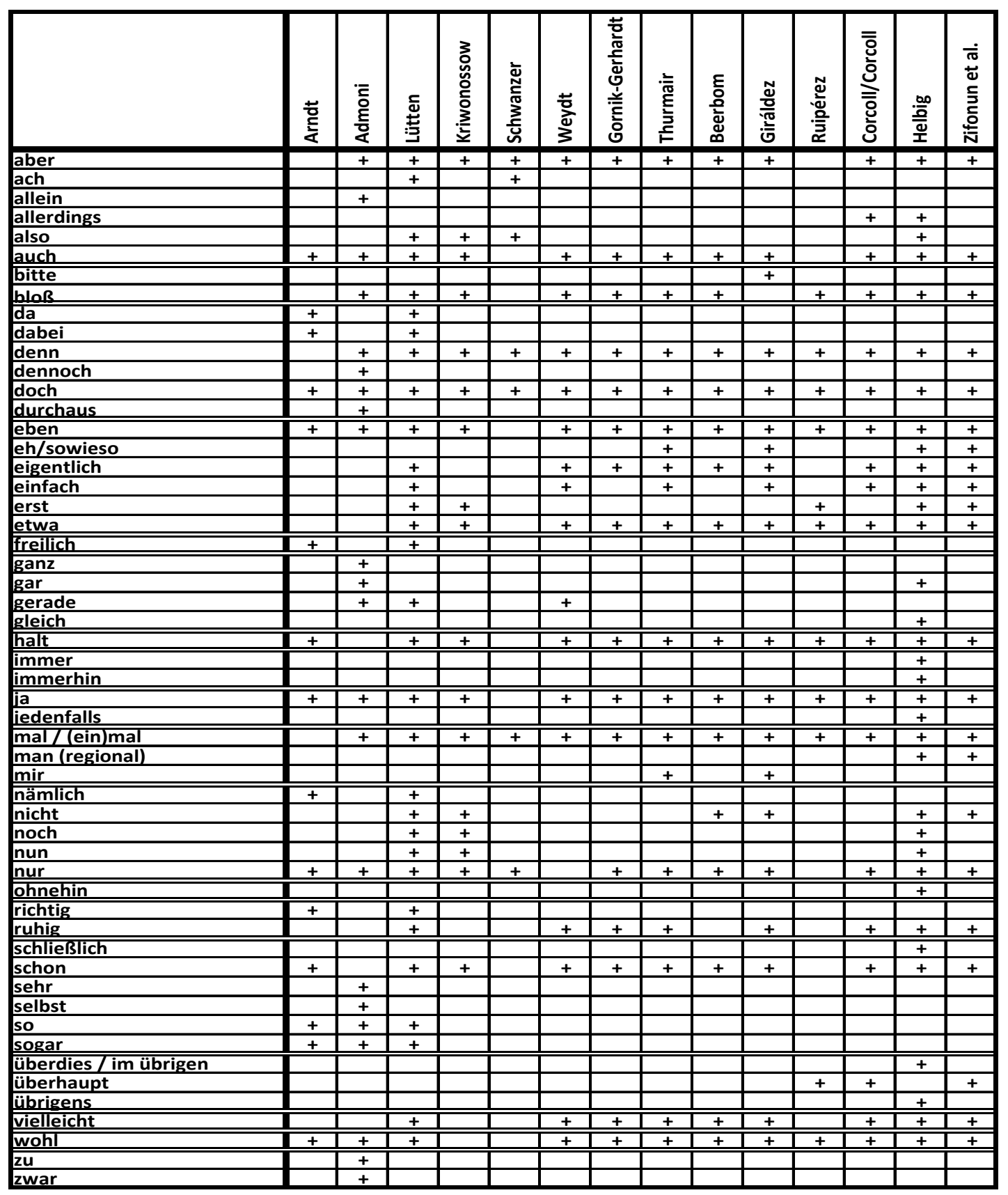

Fig. 1: Inventarios de PM 
En la tabla de la Fig. 1. se presentan a modo de ejemplo diferentes propuestas de clasificación del grupo Partikeln considerados en un sentido amplio (cf. Admoni 1970; Arndt 1960; Lütten 1977) y otros que además se centran en el grupo Modalpartikel (=cast. 'partícula modal') como subgrupo derivado del anterior (cf. Kriwonossow 1977a; Schwanzer 1965; Weydt 1969; Gornik-Gerhardt 1981; Thurmair 1989; Beerbom 1991; Giráldez 2002). Finalmente, se refieren también otros inventarios que se muestran en dos libros de gramática alemana para hispanohablantes (cf. Ruipérez 1992; Corcoll/Corcoll 1994) y en dos libros de gramática alemana (cf. Helbig 1994; Zifonun/Hoffmann/Strecker 1997). La tabla pretende proporcionar al lector una visión global y sintética además de pretender facilitar con ello que se puedan contrastar de los diferentes inventarios indicados.

En síntesis, tales perspectivas se pueden agrupar en dos grandes grupos. Por una parte, aquellas que consideran el grupo de las partículas en un sentido amplio y, por otra parte, aquellas que las consideran en un sentido más restringido. Además, la influencia del contexto comunicativo en que se enuncian las partículas parece ser un aspecto ampliamente consensuado como se verá a continuación.

Las características comunes de las propuestas son la homografía u homofonía de algunas de las partículas integrantes y la polifuncionalidad. Una misma partícula puede cumplir funciones diferentes según el contexto oracional en que se presenta y, asimismo, determinar variaciones semánticas, pero también las propias condiciones de uso de cada partícula y la dependencia del contexto comunicativo en que se emiten son criterios importantes para describir y configurar una categoría gramatical diferenciada del resto de categorías (cf. Weydt 2001: 784; Kwon 2005).

El hecho de que algunas partículas presenten formas homógrafas u homófonas en otras categorías gramaticales y que, consecuentemente, tengan la facultad de desempeñar funciones distintas se debe a la valoración subjetiva del contenido enunciativo que realiza el hablante en el momento de la emisión. Este valor añadido, dependiente del contexto gramatical en que se inserta, es el que determina el carácter modal de la partícula frente a las diferentes funciones que esta pueda desempeñar en otros contextos enunciativos $\mathrm{y}$, asimismo, permite la subclasificación como grupo de partículas específico.

Algunos autores proponen diferentes niveles de descripción de la realidad que se expresa a través de la PM o combinación de estas. Weydt (1969) propone dos niveles de expresión de la realidad. Por una parte, existiría un Darstellungsebene (=cast. 'nivel de representación'), circunscrito únicamente a la manifestación objetiva de la realidad sin expresar ningún tipo de juicio, interpretación o valoración subjetiva. Se trata únicamente de expresar la relación existente entre los conceptos e ideas que se enuncian, por lo que en este nivel solo se presenta la información de tipo verbal que el hablante cree conveniente promover. En este nivel de descripción la partícula realiza funciones de tipo adverbial o de tipo conjuntivo. En cambio, en el siguiente nivel de descripción que propone, el Intentionsebene (=cast. 'nivel de intención'), se expresa la interpretación o valoración subjetiva del hablante con respecto al contexto comunicativo en que se produce la emisión. El hablante añade un elemento emocional al contenido puramente informativo de su discurso, expresa su posicionamiento emocional en relación con su discurso utilizando otros mecanismos del código oral que van más allá de la mera verbalización de los hechos o circunstancias discursivas. A través de estos mecanismos considerados extralingüísticos, el hablante vehicula su posicionamiento emocional respecto a lo dicho en sus emisiones verbales. La entonación, la gesticulación y el paralenguaje formarían parte de este conjunto de mecanismos extralingüísticos, pero también este sería el ámbito de expresión de las PM. Es en este nivel donde la partícula aporta un significado específico, un modo particular de entender el significado general del enunciado al que acompaña (cf. Kriwonossow 1977a: 45; Weydt 1969: 61; Bublitz 1978: 6-9; Franck 
1980: 256), con lo que el nivel de análisis de tipo pragmático presentaría un marco descriptivo fundamental para la caracterización de las PM.

La expresión de la realidad, en esencia, está sujeta a un proceso cognitivo de percepción y procesamiento subjetivo, que en definitiva da como resultado diferentes modos de entender y expresar la realidad. Para Kriwonossow (1977a) -siguiendo la teoría de la modalidad desarrollada por un grupo de lingüistas soviéticos (Winogradow, Schwedowa, Michelewitsch, Budagow y Bisenieks) ${ }^{5}$ la expresión del modo en que se interpreta la realidad se puede realizar, en primer lugar, de forma objetiva -que se corresponde con lo que unos años más tarde Weydt (1969) denomina nivel de representación referido anteriormente-. Los diferentes grados de significación de la interpretación objetiva de la realidad se expresan a través de la propia ordenación sintáctica de los elementos que la integran y también por la modalidad entonativa, es decir, la entonación que caracteriza los enunciados de tipo interrogativo, declarativo y exclamativo. De esta manera, se determinan diferentes tipos de modalidad: una modalidad interrogativa, otra modalidad declarativa $\mathrm{y}$, finalmente, una modalidad exclamativa. En segundo lugar, refiere un modo de expresión subjetivo -al que Weydt (1969) denomina nivel de intención-, que explicaría el valor añadido que el hablante expresa en un determinado enunciado. Esta valoración subjetiva se distingue en los diferentes niveles de significación en los enunciados de tipo interrogativo, declarativos y exclamativos. Así, un enunciado interrogativo, por ejemplo, presentaría variantes entonativas según el nivel de expresión de la modalidad en que se enmarque. Además, ambos tipos de modalidad -objetiva y subjetiva- o ambos tipos de significación -modal-objetiva y modal-subjetiva, respectivamente- son interdependientes, es decir, son dos aspectos de un mismo fenómeno que coexisten en el enunciado (cf. Kriwonossow 1977a: 306). De esta forma, es posible establecer una asociación entre modalidad oracional y PM hasta tal punto que parece imposible deshacer el binomio modalidad oracional-PM. (cf. ibd.: 51). Finalmente, Kriwonossow (ibd.: 307) concluye que la relación que se establece entre PM y entonación es tan estrecha que ambos elementos presentan la misma finalidad, que no es otra que poner de manifiesto el significado modal-subjetivo de la oración.

Sobre este último aspecto indicado creemos que es interesante destacar que el primer autor que estudia las PM desde un enfoque oral incide en la relación existente entre estos elementos especialmente característicos de la lengua alemana y su manifestación predominante a través de códigos orales como la lengua espontánea y la entonación. Sin embargo, cabe señalar también que Kriwonossow (ibd.: 330) basa su análisis de la entonación en un corpus de datos extraído de 15 fuentes literarias, pero precisa que el significado modal-subjetivo no solo se expresa principalmente mediante la entonación, sino que las PM están tan estrechamente vinculadas con la entonación que a través de ellas también se expresa el significado modalsubjetivo porque entonativamente el verbo y la PM forman una unidad fonética (phonetisches Wort (=cast. 'palabra fonética')) con un único acento que recaería evidentemente sobre el verbo. Esta relación, sin embargo, ha sido muy poco desarrollada tras 50 años de investigación, teniendo en cuenta los numerosos trabajos que se han derivado desde la investigación de Kriwonossow en 1963.

Otro aspecto importante a tener en cuenta es el carácter eminentemente oral ${ }^{6}$ de las partículas en general y de las PM de forma específica. Las partículas se manifiestan principalmente en el ámbito oral de la lengua (cf. Kriwonossow 1977a; Weydt 1969; Helbig 1994; Hentschel/Weydt 1989; Beerbom 1991; Kwon 2005, entre otros) y su baja frecuencia de aparición en documentos escritos, tanto en textos de tipo literario, como en transcripciones

\footnotetext{
${ }^{5}$ Autores y escritos respectivos se aluden expresamente en Kriwonossow (1977a: 56, n. a. p. no 1).

${ }^{6}$ Helbig (1994: 11-12) señala que la manifestación de partículas en lengua escrita es bastante más escasa que en lengua oral.
} 
escritas de manifestaciones orales caracterizadas por un grado de espontaneidad relativamente elevado (cf. Lütten 1977; Kunow 1997; Vural 2000), se revela mayoritariamente asociada a una tipología textual que simula la estructura dialógica de la interacción de un interlocutor o entre dos o más interlocutores. (cf. Hentschel/Weydt 1989: 14).

En definitiva, a partir del momento en que se centra el interés en analizar el ámbito oral de la lengua, las PM adquieren un especial relieve. En comparación con la lengua escrita la presencia de PM parece ser predominante en lengua oral ${ }^{7}$. Por otra parte, el interés centrado en la oralidad y en las PM en particular propicia una cantidad ingente de estudios comparativos en los que se examina la posible equivalencia de las PM no solo con otros elementos lexemáticos propios de las lenguas que se comparan, sino que también se sugieren otro tipo de mecanismos de expresión de la modalidad (entonación, elementos morfemáticos, gestualidad). Estos estudios comparativos no solo se han llevado a cabo con lenguas pertenecientes a la familia lingüística germánicas ${ }^{8}$ con un inventario de PM y frecuencia de uso variable, sino también con otras lenguas pertenecientes a familias lingüísticas diferentes de la germánica, por ejemplo, con las lenguas románicas ${ }^{9}$; con la rama túrcica de las lenguas altaicas (cf. Vural 2000, con el turco); con las lenguas eslavas ${ }^{10}$; con las lenguas ugrofinesas (cf. Liefländer-Koistinen 1989, con el finés) y con lenguas orientales ${ }^{11}$.

\section{Descripción lingüística y terminología}

Desde un punto de vista descriptivo, las PM han presentado y presentan un problema clasificatorio, no solo desde un punto de vista morfológico, sino también a nivel sintáctico, semántico, pragmático y suprasegmental, ya que, como ya se ha mencionado anteriormente, pueden presentar formas homónimas y homófonas en otras categorías gramaticales y además de tener la posibilidad de desempeñar funciones distintas.

Por ello, desde que las PM adquieren interés como ámbito de investigación lingüística, se inicia un proceso de clasificación con el fin de establecer de forma unívoca las características demarcativas que distinguirían este grupo de palabras de otras categorías gramaticales adverbios, conjunciones, preposiciones, adjetivos-.

Esta situación ha dado lugar a diferentes puntos de vista que, a su vez, derivan en proposiciones diversas para la delimitación y denominación de las PM como grupo homogéneo y diferenciado. Como consecuencia, se obtiene una intricada, discordante y confusa red terminológica tanto para el grupo Partikeln considerado en sentido amplio como para el grupo de PM y que, lejos de presentar un ámbito de estudio concreto, definido y uniforme, dificulta aún más la investigación por la ausencia de consenso unitario, tanto a nivel terminológico como a nivel de criterios clasificatorios (cf. Kriwonossow 1977a; Beerbom 1991). De esta forma, dependiendo de los diferentes enfoques con que se emprenden las diferentes propuestas descriptivas, se proponen diferentes términos para denominar este grupo de partículas.

\footnotetext{
7 Cf. Kriwonossow 1977a: 6; Weydt 1969: 9 y 2001: 783; Helbig/Buscha 1993: 477; Helbig 1994: 11-12; Lütten 1977: 79; Thurmair 1989; Nehls 1989: 282; Beerbom 1991: 22-23; Heggelund 2001 y Schlicker 2003: 184 entre otros.

${ }^{8}$ Cf. Asbach-Schnitker 1977; O'Sullivan/Rösler 1989 y Nehls 1989, con el inglés; cf. Westheide 1989 y Foolen 2003, con el neerlandés; cf. Heinrichs 1981 y Aijmer 1996, con el sueco; y cf. Heggelund 2001, con el noruego.

${ }^{9}$ Cf. Weydt 1969 y Dalmas 1989 con el francés; cf. Beerbom 1991, con el castellano; cf. Harden 1989; Franco 1989; Schmidt-Radefeldt 1989; Kunow 1997; Nunes 2012 y Aquino 2012, con el portugués; cf. Ulrich 1989 y Stănescu 1989, con el rumano.

${ }^{10}$ Cf. Ličen 1989, con el serbocroata; cf. Nekula 1996, con el checo; cf. Korošec 1999, con el esloveno; y cf. Kaţny 1989 , con el polaco.

${ }^{11}$ Cf. Sekiguchi 1977; Kawashima 1989; Kosaka 1989 y Werner 1998, con el japonés; cf. Choi 1989, con el coreano.
} 
En el primer apartado se expusieron diferentes términos que los lingüistas adscritos a la normativa estilística proponían para calificar el conjunto de PM. Para poner de manifiesto la complejidad terminológica relacionada con este grupo de palabras, se exponen a continuación otros términos utilizados para referirse a estos elementos y que podemos encontrar en la literatura científica sobre el tema.

Así, podemos observar diferentes denominaciones que tienen que ver con la concepción de que a través de las PM se representa el punto de vista de los interlocutores con respecto al desarrollo discursivo. De esta forma, se proponen designaciones tales como intentionale Partikeln (=cast. 'partículas intencionales') (cf. Rall 1981); Existimatoren (=cast. 'estimadores') (cf. Thun 1984); Einstellungspartikeln (=cast. 'partículas actitudinales') (cf. Doherty 1987); epistemic particles (=cast. 'partículas epistémicas') (cf. ibd.). Otras denominaciones tienen que ver más con el significado emotivo que se intenta expresar, obteniendo de esta forma términos tales como emotional-expressive Partikeln (=cast. 'partículas emocional-expresivas') (cf. Malige-Klappenbach 1981); modale Partikeln (=cast. 'partículas modales') (cf. Kriwonossow 1977a; Adler 1964; Brinkmann 1971: 499); modale oder emotional-expressive Partikeln (=cast. 'partículas modales o partículas emocionalexpresivas') (cf. Erben 1958); modal-expressive Partikeln (=cast. 'partículas modalexpresivas') (cf. Lütten 1977: 79). Otros autores opinan que las PM tienen una clara función comunicativa y por este motivo las denominan kommunikative Partikeln (=cast. 'partículas comunicativas') (cf. Rath 1975: 222); Verknüpfungspartikeln (=cast. 'partículas conectoras') (cf. Becker 1976); Konsensus-Konstitutiva (=cast. 'partículas constituyentes de consenso o acuerdo') (cf. Lütten 1977: 326, 1979); Gesprächwörter (=cast. 'palabras conversacionales') (cf. Burkhardt 1982); o illokutive Indikatoren (=cast. 'indicadores ilocutivos') (cf. Helbig 1977).

En síntesis, parece existir consenso en que la inflexibilidad correspondería a la característica morfológica principal como criterio clasificatorio dentro del grupo Partikeln. Sin embargo, este criterio, que, por una parte, diferenciaría claramente al grupo Partikeln de otras categorías gramaticales como verbos, adjetivos, nombres, pronombres y artículos, por otra parte, haría difícil establecer diferencias significativas entre este grupo y, por ejemplo, el de los adverbios, las preposiciones y las conjunciones, que también formarían parte del grupo de categorías agrupadas bajo el criterio de inflexibilidad. Se ha pretendido proporcionar una identidad lingüística específica y diferenciada del resto de categorías, pero este criterio morfológico aglutina en realidad un conjunto heterogéneo de palabras y no parece ser suficiente para establecer una delimitación clara como categoría gramatical diferenciada (cf. Lütten 1977: 126; Helbig 1994:19) y, por tanto, deja abiertas las cuestiones de homografía y homofonía por un lado, y polifuncionalidad por otro, convirtiéndose en características específicas de este grupo de palabras.

Se inicia entonces un proceso de subclasificación del grupo Partikeln teniendo en cuenta las diversas funciones que pueden realizar según el contexto comunicativo, aunque este proceso tampoco está exento de dificultades. En primer lugar, se toma en consideración la característica de inflexibilidad o indeclinabilidad como criterio básico para realizar una primera diferenciación, que, como se ha indicado más arriba, es insuficiente. En segundo lugar, los diferentes términos que se clasifican dentro del grupo Partikeln también se consideran de igual forma en otras lenguas diferentes del alemán, como por ejemplo en ruso, en inglés y en las lenguas románicas, pero estas otras lenguas además consideran también dentro de este grupo otra serie de elementos morfemáticos como los prefijos y los sufijos e incluso interjecciones tales como huch, pfui, ojemine y elementos paralingüísticos como $\mathrm{mhm}$, $h m$ o $m h-m h$ y hmm-hmm, que, sin embargo, en alemán pertenecerían al grupo de las interjecciones (cf. Kriwonossow 1977a: 31; Beerbom 1991: 51; Hentschel/Weydt 1989: 4, 15; y Weydt 2001: 791-792). Por tanto, desde esta perspectiva, este criterio morfológico, que 
permite inicialmente acotar las posibilidades clasificatorias del grupo Partikeln, no solo no resuelve la problemática existente, sino que además presenta diferencias terminológicas significativas en comparación con otras lenguas. Es por este motivo que tal criterio parece ser inapropiado o insuficiente para determinar y clasificar las PM, ya que, de esta forma, el debate abierto en la descripción y clasificación del grupo Partikeln quedaría circunscrito de forma exclusiva al ámbito lingüístico germánico.

Llegados a este punto, resulta necesario explorar otros dominios para poder establecer una subclasificación concreta de las características del grupo Partikeln. Por ello, se plantea otro enfoque descriptivo basado en las relaciones sintácticas que se establecen con otros componentes oracionales.

Desde esta perspectiva, uno de los criterios ampliamente consensuado que diferenciaría al grupo Partikeln en general y al grupo de PM en particular de otras categorías gramaticales como adverbios y conjunciones es que las partículas no podrían ocupar el primer lugar en la oración, esto es, en el llamado Vorfeld (=cast. 'precampo'), precediendo inmediatamente a la forma conjugada del verbo que ocupa generalmente la segunda posición, mientras que los adverbios o complementos adverbiales sí pueden situarse en esta parte de la oración. Esta característica lleva a determinar que no pueden desempeñar de forma aislada ningún tipo de función sintáctica, sino que formarían parte integral de una estructura sintagmática, la cual sí que desarrollaría funciones sintácticas en la oración. En consecuencia, las partículas no pueden ser consideradas como elementos gramaticales independientes con funciones sintácticas definidas (cf. Helbig 1994: 21). Sin embargo, también se observan discrepancias con respecto a este criterio en Engel (1996: 17-19) que sí las considera "con capacidad para ocupar el primer lugar de la oración"12 o "con capacidad para posicionarse en el precampo de la oración"13.

A partir de estas características, que diferenciarían las partículas de los adverbios, de las conjunciones y de las preposiciones, se pueden determinar otras características como la imposibilidad de poder ser utilizadas como respuesta a las Entscheidungsfragesätze (=cast. 'oraciones interrogativas absolutas') o a las Ergänzungsfragesätze (=cast. 'oraciones interrogativas pronominales'), facultad que sí pueden desempeñar las Modalwörter (=cast. 'palabras modales') o también los Satzadverbien (=cast. 'adverbios de frase') como supuestamente, confiadamente, posiblemente, afortunadamente, gracias a Dios, por desgracia, a través de las cuales el hablante expresa una valoración subjetiva o emocional sobre un hecho circunstancial ${ }^{14}$. Otras categorías que podrían desempeñar esta función son los adverbios y las denominadas Antwortpartikeln ${ }^{15}$ (=cast. 'partículas de respuesta'), así como también las formas adverbiales homógrafas u homófonas de las PM como ja, nein, doch (cf. Weydt 1969: 67; Hentschel/Weydt 1989: 4; Beerbom 1991: 27).

Las características generales del grupo Partikeln son aplicables a cada subgrupo de partículas en que se divide-Modalwörter o Satzadverbien; Gradpartikeln o Intensivpartikeln o también

\footnotetext{
12 Del término original en alemán erststellenfähig.

${ }^{13}$ Del término original en alemán vorfeldfähig.

${ }^{14}$ Cf. Bußmann (2008: 602): "vermutlich, hoffentlich, möglicherweise (...) glücklicherweise, gottlob, leider". Indican el grado de probabilidad de una declaración. En algunas gramáticas, este grupo de palabras se considera como un grupo diferenciado e independiente (cf. Helbig/Buscha 1993; Admoni 1970).

15 Existe una curiosa ambigüedad terminológica para designar este grupo de palabras: Antwortpartikeln $(=$ cast. 'partículas de respuesta') - partículas afirmativas y partículas negativas con función adverbial- (cf. Helbig 1988/1994: 30; Hentschel/Weydt 1989: 15; y en Weydt 2001: 791-792, donde, además, se incluyen Interjektionen (=cast. 'interjecciones'): $m h m, h m, m h$-mh o hmm-hmm, esto es, los Discourse markers de Shiffrin (1987), los Discourse signals de Stenström (1989), o los Alternantes de Poyatos (2004); pero también tienen cabida manifestaciones de tipo no verbales, como ciertos movimientos realizados con la cabeza (verticales y horizontales) o indicaciones efectuadas con las manos o con los dedos (cf. Weydt 2001).
} 
Fokuspartikeln; Negationspartikeln; Vergleichspartikeln; Antwortpartikeln; Modalpartikeln (Abtönungspartikeln), Situativpartikeln-16, aunque cada subgrupo presenta diferencias mínimas entre sí según el significado, la función y el contexto en que aparecen, resulta difícil establecer claramente el inventario de partículas que integra cada subgrupo.

Centrándonos exclusivamente en el subgrupo de PM, se examinan a continuación las características que son aplicables mayoritariamente y de forma consensuada a este grupo de palabras. Por una parte, se pretende ofrecer al lector una síntesis pormenorizada obtenida de diversas fuentes de los aspectos característicos de este grupo de palabras en diferentes niveles lingüísticos. Por otra parte, se quiere mostrar con ello el elevado grado de complejidad que supondría establecer con certeza un listado de palabras que pudiera delimitarse y diferenciarse de otras categorías gramaticales.

Una de las características más recurrentes es que las PM se encuentran siempre precediendo de forma inmediata al elemento remático de la oración, lo cual significa que su ubicación se puede establecer en el Mittelfeld (=cast. 'intercampo oracional'), y próxima a la forma verbal conjugada. Sin embargo, se puede dar el caso de que el elemento remático de la oración ocupe el primer lugar, delante del verbo conjugado, o que este elemento esté formado por el verbo. En estos casos, la PM suele aparecer ocupando posiciones finales en la oración (cf. Hentschel 1986; Hentschel/Weydt 1989) y, en todo caso, delante del adverbio nicht (cf. Beerbom 1991).

Otras características de las PM consisten en que, por una parte, no pueden ser transformadas en una oración, como sucede con las palabras modales (=al. 'Modalwörter') referidas más arriba. Por otra parte, parece haber un criterio bastante consensuado en asociar determinadas PM con determinados tipos de frase o modalidades oracionales (cf. Kriwonossow 1977a; Weydt 1969; Hentschel/Weydt 1989, 2013; Beerbom 1991; Kwon 2005).

En base a estos criterios ampliamente consensuados, se determina que las partículas no contienen un valor léxico independiente ${ }^{17} \mathrm{y}$, en consecuencia, el valor semántico que aportan al significado proposicional es nulo, ya que no desempeñan ningún tipo de función sintáctica porque no pueden ser consideradas elementos independientes. Es por este motivo que su uso podría ser considerado facultativo, porque la omisión, inicialmente, no afecta ni a la estructura sintáctica de la oración, ni a la gramaticalidad de la frase (cf. Helbig 1994: 24; Ruipérez 1992: 187), como tampoco a la modalidad oracional del enunciado en que se insertan (cf. Kriwonossow 1977a: 59). Sin embargo, precisamente por el carácter facultativo, es decir, por la posible omisión o no omisión de la partícula, se pueden determinar diferencias en el plano semántico-pragmático, es decir, los diferentes significados que pueden ser atribuibles al enunciado dependiendo de la presencia o ausencia de partícula (cf. Admoni 1970; Weydt 1969, 2001; Bublitz 1978; Franck 1980; Helbig 1994; Ruipérez 1992; Corcoll/Corcoll 1994).

Igualmente, la presencia o ausencia de una determinada PM o una determinada combinación de PM puede estar asociada a un determinado tipo de modalidad oracional (cf. Ferrer 2010: 95) $y$, por esto, en función del contexto oracional en que aparecen las PM, se puede determinar el tipo de significado modal que aporta una determinada partícula o combinación de partículas. Así, se llega a establecer que el ámbito de influencia de la PM se da a nivel sintagmático y también a nivel oracional. Sin embargo, este criterio no permite distinguir

\footnotetext{
16 Traducción al castellano: palabras modales, adverbios de frase; partículas de grado o partículas de intensidad o también partículas de foco; partículas de negación; partículas de comparación; partículas de respuesta; partículas modales (partículas matizadoras); partículas de situación.

17 Lutzeier (1985: 21, citado en Schlicker 2003: 185) sostiene que contienen un valor léxico definido e independiente, $\mathrm{y}$, consecuentemente, las PM desempeñarían funciones sintácticas determinadas, ya que su uso en un determinado enunciado implica la expresión de un significado general distinto en comparación con el mismo enunciado sin partícula. Por ello, reciben también el nombre de palabras completas (=al. 'Vollwörter'), palabras con significado (=al. 'Bedeutungswörter'), (palabras) autosignificadoras (=al. 'Autosemantika').
} 
claramente entre las diferentes funciones que pueden desempeñar las partículas con formas homónimas u homófonas en otras categorías gramaticales ni cuándo actúan como PM.

Para finalizar este apartado, se expone un resumen de los rasgos que mayoritariamente caracterizan a las PM. Por tanto, la complejidad de las PM determina que no puedan ser definidas a partir de un único nivel lingüístico, sino que su sistematización lingüística abarque prácticamente todos los niveles descriptivos:

\section{Rasgos morfo-sintácticos:}

- No se pueden flexionar (no se pueden conjugar ni se pueden declinar ni se pueden comparar) (cf. Weydt 1969: 67-68; Gornik-Gerhardt 1981: 33; Helbig 1994: 32; Kosaka 1989: 161; Stănescu 1989: 268; Werner 1998: 95; Heggelund 2001; Giráldez 2002: 63). Sin embargo, Wegener (1989: 56) sostiene que las formas declinadas en dativo de los pronombres personales en singular ich y $d u$, esto es, mir y dir, y las respectivas formas en plural wir y ihr, esto es, uns y euch, que no realizan la función de complemento indirecto, sino en calidad de dativo ético, actúan como PM. Para Giráldez (2002: 61): "Aunque se trata de un lexema flexionado (mir, dir y uns), en la función de PM carece de flexión. Además, si mir, dir y uns son átonas, no podrán aparecer en el precampo verbal de la oración, sino que les corresponderá un lugar en el campo verbal."

- Presentan formas homógrafas u homófonas en otras categorías gramaticales (cf. Weydt 1969: 68; Lütten 1977: 393; Gornik-Gerhardt 1981: 33; Helbig 1994: 32; Kosaka 1989: 161; Beerbom 1991: 26; Zifonun/Hoffmann/Strecker 1997: 1209; Werner 1998: 95; Heggelund 2001; Giráldez 2002: 49).

- Las PM son por regla general monosilábicas (cf. Arndt 1960: 326; Bublitz 1978: 37; Gornik-Gerhardt 1981: 33; Helbig 1994: 33; Beerbom 1991: 26; Nekula 1996: 47; Heggelund 2001). Sin embargo, palabras bisílabas como aber, eben, einfach, etwa, ruhig y vielleicht, así como palabras trisílabas como eigentlich, gerade y überhaupt también pueden ser consideradas PM.

- No pueden ser utilizadas como respuesta a un determinado tipo de pregunta (Entscheidungsfragen y Ergänzungsfragen) ${ }^{18}$, a diferencia de las Modalwörter (=cast. 'palabras modales') y los adverbios (ja, nein, doch) respectivamente (cf. Arndt 1960: 330; Weydt 1969: 67-68; Iwasaki 1977: 66; Gornik-Gerhardt 1981: 34; Hartmann 1979: 126; Helbig 1994: 32; Hentschel/Weydt 1989: 14, 2013; Wegener 1989: 58; Kosaka 1989: 161; Stănescu 1989: 268; Beerbom 1991: 27; Nekula 1996: 46-47; Werner 1998: 95; Korošec 1999: 6; Heggelund 2001; Giráldez 2002: 63).

- No desempeñan una función independiente dentro de la estructura sintáctica de la frase (cf. Weydt 1969: 68; Gornik-Gerhardt 1981: 34; Ruipérez 1992: 188; Nekula 1996: 46; Korošec 1999: 6; Vural 2000: 15), sino que forman parte de una estructura sintagmática, que sí cumple un determinada función sintáctica, esto es, forman junto con el verbo una unidad entonativa independiente (cf. Kriwonossow 1977a: 242,307; Kriwonossow 1977b: 190; Iwasaki 1977: 66; Helbig 1994: 32). A este respecto, Werner (1998: 95) opina que las PM ni tienen una función gramatical independiente dentro de la estructura oracional, ni tampoco forman parte integrante de una estructura sintagmática. Diewald (2006: 423) opina que sí presentan funciones gramaticales genuinas y que su función es fundamentalmente de carácter indicial.

\footnotetext{
18 Traducción al castellano: 'oraciones interrogativas absolutas' y 'oraciones interrogativas pronominales'.
} 
- Al no realizar una función sintáctica independiente, tampoco pueden aparecer antepuestas a la emisión de la forma verbal, esto es, ocupar el primer lugar de oración (cf. Arndt 1960: 330; Weydt 1969: 67-68; Iwasaki 1977: 65; Gornik-Gerhardt 1981: 33-34; Hartmann 1979: 126; Helbig 1994: 32; Hentschel/Weydt 1989: 14, 2013; Kriwonossow 1989: 34; Wegener 1989: 57; Stănescu 1989: 268; Ruipérez 1992: 188; Nekula 1996: 47; Zifonun/Hoffmann/Strecker 1997: 1208; Werner 1998: 95; Korošec 1999: 6; Heggelund 2001; Giráldez 2002: 57; Foolen 2003; Diewald 2006: 408). Sin embargo, Kwon (2005: 16) matiza esta aserción de tal forma que determinadas PM en determinadas condiciones sintácticas sí que pueden aparecer en el precampo verbal o (=al. 'Vorfeld').

- Su posición en la oración aparece antepuesta al rema de la frase. (cf. Kriwonossow 1977a: 314; Kriwonossow 1977b: 187-188; Hentschel 1986: 236; Hentschel/Weydt 1989: 14, 2013; Kriwonossow 1989: 33; Nekula 1996: 47). Sin embargo, Werner (1998: 95) muestra discrepancias sobre este criterio al cuestionar que las PM tienen que aparecer o manifestarse delante del elemento remático de la oración.

- Las PM aparecen generalmente detrás de la forma conjugada del verbo, esto es, en el intercampo de la oración (=al. 'Mittelfeld') (cf. Arndt 1960: 330; Kriwonossow 1977a: 307; Bublitz 1978: 36-37; Franck 1980: 20; Gornik-Gerhardt 1981: 35; Hentschel 1986; Kriwonossow 1989: 34; Wegener 1989: 58; Kosaka 1989: 161; Nekula 1996: 47; Werner 1998: 95; Giráldez 2002: 57; Foolen 2003; Hentschel/Weydt 2013).

- Las PM se encuentran como las Modalwörter (=cast. 'palabras modales') ante nicht (cf. Gornik-Gerhardt 1981: 35; Helbig 1994: 34; Beerbom 1991: 27; Stănescu 1989: 268) y las unidades lingüísticas que se encuentran entre la forma verbal conjugada y la/-s partícula/-s modal/-es son siempre átonas (cf. Kriwonossow 1977a: 314; GornikGerhardt 1981: 35; Helbig 1994: 33).

- Las PM, a diferencia de las Modalwörter (=cast. 'palabras modales'), no pueden transformarse en una oración. No se pueden parafrasear ${ }^{19}$ o sí que son parafraseables sin que ello suponga la modificación proposicional (cf. Nekula 1996: 47) o para comprender el valor significativo que aportan (cf. Helbig/Kötz 1981: 34; Hentschel/Weydt 2013) o para explicar o aclarar la función que desempeñan en determinados tipos de enunciados (cf. Beerbom 1991: 42-43) o como un recurso útil en las traducciones a otras lenguas (cf. Albrecht 1977: 32-35).

- Las PM se pueden combinar, aunque la combinación de dos o más PM parece depender de ciertas restricciones distributivas ${ }^{20}$. Werner (1998: 95) es de la opinión de que las PM no solo se pueden combinar entre sí, sino que además también pueden combinarse con otros modificadores ilocutivos.

- Las PM tienen carácter facultativo, esto es, que su omisión no afecta a la gramaticalidad de la construcción oracional ${ }^{21}$. Sin embargo, Steinmüller (1981: 139) es de la opinión de que el uso de PM en los intercambios comunicativos entre inmigrantes turcos y nativos berlineses es obligatorio, ya que de lo contrario no solo se vulnera la comprensión sino la determinación de significados claramente reconocibles.

19 Cf. Arndt 1960: 330; Kriwonossow 1977a: 11-12; Iwasaki 1977: 65-66; Gornik-Gerhardt 1981: 34; Hartmann 1979: 126; Giráldez 2002: 64.

20 Cf. Helbig/Buscha 1993: 498; Bastert 1985: 40-41; Thurmair 1989; Hentschel/Weydt 1989: 14, 2013; Heggelund 2001; Lemnitzer 2001; Giráldez 2002: 63.

21 Cf. Reiners 1961: 339-340; Arndt 1960: 331; Franck 1980: 21; Helbig 1994: 33; Ruipérez 1992: 187; Nekula 1996: 47; Werner 1998: 95; Korošec 1999: 7; Giráldez 2002: 57; Kwon, 2005: 19-22. 
Continuando en esta misma línea, Diewald (2006: 423) afirma que se trata de elementos indispensables en la interacción hablada.

\section{Rasgos semántico-pragmáticos:}

- No poseen un significado léxico independiente, sino que lo adquieren a través de la relación con los demás elementos que componen la oración ${ }^{22}$. Sin embargo, Lütten (1977: 112) cuestiona el carácter adicional o facultativo de las PM como elementos lexemáticos e induce la posibilidad de que las PM desempeñen una función independiente.

- El valor significativo de las PM está sujeto a una fuerte dependencia del contexto comunicativo $^{23}$.

- Las funciones de la partícula dependen en parte del significado etimológico. Por ejemplo, aber puede comportarse como la conjunción adversativa pero, pero también como PM (cf. Beerbom 1991: 26; Vural 2000) o en relación con las PM halt/eben (cf. Nekula 1996: 145-156).

- La mayoría de las PM aparecen asociadas a un determinado tipo de modalidad oracional, principalmente: declarativa o asertiva, interrogativa, imperativa $o$ exhortativa; pero también, en modalidades oracionales de tipo exclamativa y desiderativa u optativa ${ }^{24}$. Sin embargo, Werner (1998: 96) muestra discrepancias sobre este criterio al cuestionar la dependencia de las PM de una determinada modalidad oracional y del tipo de ilocución. Como contrapartida, propone que las PM no necesariamente deben estar asociadas a una determinada modalidad oracional o a un determinado tipo de ilocución, sino que más bien su manifestación es compatible con determinada modalidad oracional o con determinado tipo de ilocución (cf. Werner 1998: 130, n.a.p. $\mathrm{n}^{\mathrm{o}}$ 108). Además, sostiene que tanto la modalidad oracional como otros parámetros pragmáticos de carácter paralingüístico no se expresan a través de las PM, sino sobre todo a través de la entonación (cf. Werner 1998: 135).

- El Skopus ${ }^{25}$ de la PM es toda la oración ${ }^{26}$ o solo una parte (cf. Admoni 1970: 205). Forman conjuntamente con el verbo una unidad fonética (=al. 'phonetisches Wort') (cf. Kriwonossow 1977a: 307; Kriwonossow 1989: 33).

- A través de las PM, el hablante puede expresar diferentes puntos de vista, estados anímicos y emociones.

\footnotetext{
22 Cf. Kriwonossow 1977a: 242,307; Weydt 1969: 68; Iwasaki 1977: 66; Bublitz 1978: 38; Helbig 1994: 32; Hentschel/Weydt 1989: 14; Kriwonossow 1989: 33; Nekula 1996: 47; Heggelund 2001; Giráldez 2002: 57.

23 Cf. Kriwonossow 1977a: 314; Gornik-Gerhardt 1981: 28; Heinrichs 1981: 116; Helbig 1994: 33; Hentschel/Weydt 1989: 14; Kriwonossow 1989: 34; Ulrich 1989: 41; Heggelund 2001; Möllering 2001: 130; Giráldez 2002: 51-55; Foolen 2003; Kwon, 2005: 1.

24 Cf. Kriwonossow 1977a: 315; Weydt 1969: 27; Gornik-Gerhardt 1981: 34-35; Hartmann 1979: 125; Franck 1979: 3; Doherty 1987: 100-114; Bastert 1985: 33-35; Helbig 1994: 33; Kriwonossow 1989; Wegener 1989: 58; Kosaka 1989: 161; Nehls 1989: 283; Beerbom 1991: 27; Nekula 1996: 47; Heggelund 2001; Giráldez 2002: 64; Foolen 2003; Kwon 2005; Hentschel/Weydt 2013.

25 Término utilizado en alemán para referirse al ámbito de influencia de las PM.

26 Cf. Arndt 1960: 331; Weydt 1969: 68; Lütten 1977: 393; Iwasaki 1977: 65; Gornik-Gerhardt 1981: 34-35; Hentschel 1986; Helbig 1994: 32; Hentschel/Weydt 1989: 14; Kosaka 1989: 161; Beerbom 1991: 27; Nekula 1996: 28-29, 46; Werner 1998: 95; Heggelund 2001.
} 


\section{Rasgos suprasegmentales:}

- Normalmente, las PM son átonas, aunque presentan formas homófonas tónicas en otras categorías gramaticales ${ }^{27}$. Según Kriwonossow (1977a: 240) y Helbig (1994: 32), las PM son siempre átonas. Sin embargo, Gornik-Gerhardt (1981: 33,72); Meibauer (1994: 224), Hentschel (1986: 155) y Doherty, (1985: 100,128-138) consideran que las PM schon, denn, y ja presentan variantes semánticas caracterizadas por el rasgo [+tónico]. Además de las PM denn y ja, Doherty (1987) aprecia también variantes semánticas caracterizadas por el rasgo [+tónico] en las PM doch y wohl. Sin embargo, Werner (1998: 95) se muestra discrepante al cuestionar la valoración que se realiza sobre el criterio [ \pm tónico] de las PM.

\section{Rasgos según el ámbito de uso:}

- La manifestación de las PM se observa principalmente en lengua oral. Su aparición en documentos escritos es comparativamente muy inferior a su manifestación en el habla espontánea, quedando limitada a determinados tipos de texto, mediante los que se intenta reproducir la forma oral de la lengua, sobre todo a través de diálogos, monólogos y pasajes en los que se utiliza un estilo y registro lingüístico de tipo coloquial. No obstante, la mayoría de trabajos realizados en este campo de investigación utilizan como base para los análisis un corpus de datos proveniente de fuentes escritas de diversa índole.

\section{$3 \quad$ Entonación y partículas modales}

En este apartado se consideran diferentes contribuciones en las que se estudia la relación entre entonación y PM de una forma más extensa o detallada. Concretamente, se ha focalizado la atención en analizar el tipo de corpus en que se basan los diferentes autores para estudiar esta relación y la metodología de análisis empleada.

A pesar de que en general existe un amplio consenso en considerar que el medio natural predominante en que se manifiestan las PM corresponde al ámbito oral de la lengua y, dentro de este ámbito, especialmente al ámbito coloquial y familiar, se observa que la mayoría de los autores se esfuerzan por justificar la validez de los análisis que realizan sobre un corpus de datos escritos. Así, el corpus de datos que se emplea para realizar el análisis de la entonación procede de fuentes escritas de diverso género, pero principalmente extraído de obras literarias. Esto significa que, a partir de una fuente de datos que no aporta materia fónica, esto es, que no suena, se seleccionan enunciados para posteriormente realizar el correspondiente análisis acústico.

En general, el procedimiento de análisis de la entonación se caracteriza por ser marcadamente de tipo intuitivo, esto es, que no se emplea ningún tipo de instrumental técnico para el análisis de la señal acústica o sí que se utiliza una vez el corpus de datos ha sido previamente mediatizado por el investigador. En síntesis, el tratamiento analítico que recibe la entonación en su relación con las PM generalmente se realiza a partir de la propia competencia lingüística del investigador. La lectura individual de los enunciados, sea mentalmente o en voz alta, es el procedimiento habitual para determinar las características acústicas de este grupo de palabras $\mathrm{y}$, cuando las circunstancias lo permiten, los datos resultantes del análisis pueden ser contrastados con las emisiones o percepciones individuales realizadas por otros informantes (cf. Kriwonossow 1977a; Vural 2000; Kwon 2005; Moroni 2010). Mediante esta metodología solo sería factible realizar un hipotético análisis de la entonación y su relación con las PM a

27 Cf. Arndt 1960: 330; Weydt 1969: 25,68; Bublitz 1978: 37; Bastert 1985: 38; Hentschel/Weydt 1989: 14; Wegener 1989: 57; Stănescu 1989: 268; Beerbom 1991: 27; Nekula 1996: 46,62; Zifonun/Hoffmann/Strecker 1997: 1208; Heggelund 2001; Giráldez 2002: 56; Foolen 2003 y Kwon 2005: 11-12, entre otros. 
través de la competencia lingüística en alemán, lo cual delimita o circunscribe su estudio y análisis casi de forma exclusiva al ámbito lingüístico germánico (cf. Steinmüller 1981:139).

En la sección anterior, se ha indicado que uno de los rasgos fundamentales y ampliamente reconocido en la literatura especializada es que las PM son átonas. La determinación del rasgo [-tónico], que se determina a partir del análisis de textos escritos, es un criterio indispensable en base al cual se establece si una partícula pertenece o no al grupo de las PM. Sin embargo, al igual que ocurre prácticamente con el resto de características distintivas de este grupo de partículas, se observan posicionamientos discrepantes.

Asimismo, a partir del análisis de textos escritos, se determinan las diferentes modalidades oracionales - principalmente, las modalidades clásicas o básicas: declarativa, interrogativa e imperativa; pero, también se tienen en cuenta otros tipos de modalidades oracionales como la desiderativa y la exclamativa, y otras variantes dentro de cada modalidad oracional $\_^{28}$, de las cuales se indican incluso los diferentes patrones melódicos que las caracterizarían, abstraídos igualmente de forma intuitiva. También se observan ciertas alusiones a patrones melódicos de tipo paralingüístico y su asociación a determinadas PM, cuya interpretación fonológica solo sería posible desde un punto de vista nativo. Este sería el caso, por ejemplo, que se refiere en Gornik-Gerhardt (1981), en Franck (1980: 188) o en Graefen (2000) para el alemán o el que aporta Nekula (1996: 57) para el checo. De esta forma, a partir de la asociación que se establece entre modalidades oracionales lingüísticas y paralingüísticas y del análisis de textos escritos se atribuyen diferentes valores semánticos y pragmáticos. A partir de la percepción e interpretación individualizada que efectúa cada autor, se han realizado inventarios para cada PM según los diferentes contextos comunicativos en que se manifiesta, exponiendo la multiplicidad de significados diferentes que se derivan. Sin embargo, desde un punto de vista didáctico del alemán como lengua extranjera, resulta cuanto menos difícil para un estudiante de esta lengua atribuir el patrón melódico correspondiente en alemán a una oración que está leyendo, si previamente no se le ha descrito la forma melódica que corresponde a cada tipo de modalidad oracional. En el mejor de los casos, aplicará obviamente aquellos que conoce o con los que está familiarizado y nos encontraremos con que este estudiante leerá la oración escrita en alemán pronunciándola en castellano, por ejemplo.

En este sentido, la apropiación y el uso adecuado de las PM según las características específicas de cada contexto comunicativo, parece plantear serias dificultades a quienes no pertenecen a la comunidad lingüística germánica, cosa que redunda en buena parte en el tratamiento didáctico. Bastert (1985: 38) y Heggelund (2001, n.a.p. $\mathrm{n}^{\circ}$ 5), por ejemplo, reconocen la existencia de importantes lagunas y la necesidad de investigar la relación entre entonación y PM, así como también su incidencia en contextos educativos. Es de notar que esta última apreciación ya había sido indicada en el pasado (cf. Kriwonossow 1977a: 318; Iwasaki 1972: 105-106), ya que el alumno que aprende alemán como lengua extranjera debe enfrentarse con un catálogo de significados incontables, deducibles solo a partir de innumerables situaciones comunicativas que son susceptibles de producirse en situaciones interactivas reales.

El significado que una determinada PM o una determinada combinación de PM aporta a un enunciado solo puede ser abstraído a partir de la caracterización de los patrones o contornos entonativos, esto es, de la interpretación fonológica de los enunciados y de los márgenes de

\footnotetext{
${ }^{28}$ En Ferrer $(1999,2010)$ se pueden consultar diferentes tipos de variantes de las modalidades oracionales según diversas aportaciones que se han realizado desde el ámbito germánico y, en especial, por lo que respecta a la modalidad de tipo interrogativo y sus variantes.
} 
dispersión o alocontornos ${ }^{29}$ de cada uno de ellos. Únicamente de esta manera, se podría determinar no solo cómo se entona en alemán un enunciado declarativo, interrogativo o exhortativo, sino que también se podría determinar cómo se entona en alemán la indignación, el enfado, la contrariedad o el disgusto; cómo se entona en alemán la exclamación de entusiasmo y la defensa ante un reproche que, como se indica más arriba, alude Graefen (2000); o cómo se entona la confirmación restringida, la objeción atenuada, la apreciación o el juicio negativo, la justificación plausible, la urgencia y la argumentación a fortiori que, como también se indica más arriba, alude Gornik-Gerhardt (1981) como posibles significados de la PM schon; o cómo se entona en alemán la inmutabilidad, la inevitabilidad, o un hecho irrevocable mediante la PM eben a que hace referencia Möllering (2001: 141).

En esta línea, Steinmüller (1981) desarrolla su estudio empírico sobre la adquisición oral tanto a nivel coloquial como a nivel dialectal - de la lengua alemana por parte de inmigrantes turcos y al margen de la instrucción dirigida y controlada que se realiza en clase de lengua extranjera. Propone potenciar el uso de las PM en clase de lengua, explicitando las diferencias significativas de tipo cultural que pueden establecerse con respecto a los estudiantes de alemán como lengua extranjera a través de situaciones comunicativas que se dan o pueden tener lugar cuando se produce un contacto intercultural con nativos de habla alemana.

Entre las contribuciones que tratan la relación que se establece entre entonación y PM, dos casos que merecen ser destacados en este campo son los trabajos realizados por Ikoma (2007) y Torregrosa (2011). Ikoma (2007) investiga la relación entre entonación y PM a partir de enunciados extraídos de un corpus de datos escritos. La metodología que pone en práctica consiste en que un grupo de informantes nativos de la lengua alemana tienen que leer en voz alta los correspondientes enunciados seleccionados, aportando la entonación que, como nativos, establecerían de forma intuitiva teniendo en cuenta un determinado contexto de uso establecido inicialmente por el investigador. Las emisiones de los informantes se registran en formato digital y se analizan posteriormente mediante instrumentos de análisis acústico. Se trata, por tanto, de uno de los primeros trabajos que analizan la relación entre entonación y PM desde un punto de vista estrictamente acústico, a partir de materia fónica analizable y a través de instrumentos de análisis técnicos. Sin embargo, teniendo en cuenta que el desarrollo de esta investigación se realiza bajo condiciones de laboratorio, el factor espontaneidad de las emisiones queda seriamente minimizado, alejándose de un uso real, genuino y espontáneo.

Torregrosa (2011) analiza la relación existente entre PM y entonación haciendo uso de un corpus de datos oral, genuino y espontáneo. El método de análisis de la entonación que se sigue se basa en el modelo Análisis Melódico del Habla (AMH) desarrollado por Cantero (2002) y que ha sido aplicado con éxito para la descripción y caracterización de los patrones entonativos y márgenes de dispersión de diferentes lenguas pertenecientes a la misma familia lingüística como también de lenguas pertenecientes a familias lingüísticas distintas. Por otra parte, la investigación que lleva a cabo este autor no se centra únicamente en examinar la relación entre PM y entonación, sino que además se estudia la relación que se establece entre PM y entonación con la gesticulación que se desarrolla durante las emisiones orales aplicando una metodología de análisis multisistémico utilizando diferentes instrumentos técnicos para el tratamiento de datos acústicos y multimedia.

En síntesis, el estudio de la relación entre entonación y PM debería centrarse en el análisis de las emisiones reales, genuinas y espontáneas realizadas a través de los diferentes medios de expresión de la oralidad, entre los cuales no solo se cuentan las características específicas de la lengua oral sin mediación escrita y las características de la entonación de los enunciados

\footnotetext{
29 Por márgenes de dispersión o alocontornos se entienden las diversas variantes entonativas que los hablantes pueden realizar de cada una de las modalidades entonativas. Tales variantes pueden ser de tipo lingüístico, expresivo, emocional y personal de cada hablante.
} 
orales, sino también la entonación paralingüística y la gestualidad coexpresiva a las emisiones vocal-verbales.

Por tanto, creemos que retomar la investigación de las PM desde un punto de vista de la estricta oralidad, esto es, a partir de un corpus de datos audiovisual, real, genuino y espontáneo, a través del cual sea posible analizarlas y estudiarlas en su ámbito de expresión natural o predominante teniendo en cuenta los diferentes códigos orales, permitiría dar respuesta a las cuestiones que actualmente permanecen abiertas y aportaría soluciones didácticas que ayuden en la enseñanza/aprendizaje del alemán como lengua extranjera, así como también en el campo de la traducción e interpretación.

\section{Bibliografía}

Adler, Hans G. (1964): "Füllwörter". Muttersprache 74: 52-55. Referencia en Lütten 1977: 83.

Admoni, Vladimir G. ( $\left.{ }^{3} 1970\right):$ Der deutsche Sprachbau. München: Beck. (= Handbücher für das Studium der Germanistik).

Aijmer, Karin (1996): "Swedish modal particles in a contrastive perspective". Language Sciences 18: 393-427.

Albrecht, Jörn (1977): "Wie übersetzt man eigentlich eigentlich". En: Weydt, Harald (ed.): Aspekte der Modalpartikeln. Studien zur deutschen Abtönung. Tübingen, Niemeyer: 19-37.

Aquino, Marceli (2012): A função dinâmica das partículas modais alemãs doche jano ensino de línguas. Brasil: Universidade Federal de Minas Gerais. Memoria de Máster. http://hdl.handle.net/1843/LETR-8ST23Y, consultado 20 de marzo, 2014.

Arndt, Walter (1960): "'Modal particles' in Russian and German". Word 16: 323-336. Referencia en Kriwonossow 1977a.

Asbach-Schnitker, Brigitte (1977): "Die Satzpartikel wohl. Eine Untersuchung ihrer Verwendungsbedingungen im Deutschen und ihrer Wiedergabemöglichkeiten im Englischen". En: Weydt, Harald (ed.): Aspekte der Modalpartikeln. Studien zur deutschen Abtönung. Tübingen, Niemeyer: 38-61.

Bastert, Ulrike (1985): Modalpartikel und Lexikographie. Eine exemplarische Studie zur Darstellbarkeit von "doch" im einsprachigen Wörterbuch. Tübingen: Niemeyer. (= Reihe germanistische Linguistik 58).

Becker, Norbert (1976): "Die Verknüpfungspartikeln denn, mal, doch und andere". Zielsprache Deutsch 7/3: 6-12.

Beerbom, Christiane (1991): Modalpartikeln als Übersetzungsproblem. Eine kontrastive Studie zum Sprachenpaar Deutsch-Spanisch. Frankfurt a. M. etc.: Peter Lang. Tesis Doctoral publicada. (= Heidelberger Beiträge zur Romanistik 26).

Brinkmann, Hennig ( $\left.{ }^{2} 1971\right)$ : Die deutsche Sprache: Gestalt und Leistung. Düsseldorf: Schwann. Referencia en Lütten 1977: 82.

Bublitz, Wolfram (1978): Ausdrucksweisen der Sprechereinstellung im Deutschen und Englischen. Untersuchungen zur Syntax, Semantik und Pragmatik der deutschen Modalpartikeln und Vergewisserungsfragen und ihrer englischen Entsprechungen. Tübingen: Niemeyer. (= Linguistische Arbeiten 57).

Burkhardt, Arnim (1982): "Gesprächswörter. Ihre lexikologische Bestimmung und lexikographische Beschreibung". En: Mentrup, Wolfgang (ed.): Konzepte zur Lexikographie. Studien zur Bedeutungserklärung in einsprachigen Wörterbüchern. Tübingen, Max Niemeyer: 138-171.

Bußmann, Hadumod ( $\left.{ }^{4} 2008\right)$ : Lexikon der Sprachwissenschaft. Stuttgart: Kröner.

Cantero, Francisco J. (2002): Teoría y análisis de la entonación. Barcelona: Edicions de la Universitat de Barcelona. 
Colditz, Siegfried (1966): "Einige Wörter in ihrer vielseitigen Verwendbarkeit". Deutsch als Fremdsprache 1: 49-52.

Corcoll, Brigitte/Corcoll, Roberto (1994): Programm. Alemán para hispanohablantes. Barcelona: Herder.

Choi, Kyu-Ryun (1989): "Schon und noch und ihre koreanischen Entsprechungen imi und ajik". En: Weydt, Harald (ed.): Sprechen mit Partikeln. Berlin, Walter de Gruyter:129-140.

Dalmas, Martine (1989): "Sprechakte vergleichen: ein Beitrag zur deutsch französischen Partikelforschung". En: Weydt, Harald (ed.): Sprechen mit Partikeln. Berlin, Walter de Gruyter: 228-239.

Diewald, Gabriele (2006): "Discourse particles and modal particles as grammatical elements". En: Fischer, Kerstin (ed.): Approaches to discourse particles. Amsterdam, Elsevier: 423-425.

Doherty, Monika (1987): Epistemic meaning. Berlin/Heidelberg: Springer-Verlag. (= Springer series in language and communication 21).

Engel, Ulrich (1996): Deutsche Grammatik. Heidelberg: Groos.

Erben, Johannes (1958): Abriss der deutschen Grammatik. Berlin: Akademie-Verlag.

Ferrer, Hang (1999): "El modo oracional interrogativo en la lingüística germánica: rasgos formales y función, taxonomías y subtipos". Revista de Filología Alemana 7: 239-271. http://revistas.ucm.es/index.php/RFAL/article/view/RFAL9999110239A, consultado 20 de marzo, 2014.

Ferrer, Hang (2010): "Criterios para el estudio de la modalidad en alemán y en español. Los modos oracionales y las palabras modales". Revista de Filología Alemana Anejo II: 81-98. http://revistas.ucm.es/index.php/RFAL/article/view/36534, consultado 20 de marzo, 2014.

Foolen, Ad (2003): "Niederländisch toch und Deutsch doch: Gleich oder doch nicht ganz?". Linguistik Online 13. http://www.linguistik-online.de/13_01/foolen.html, consultado 20 de marzo, 2014.

Franck, Dorothea (1979): "Abtönungspartikeln und Interaktionsmanagement. Tendenziöse Fragen". En: Weydt, Harald (ed.): Die Partikeln der deutschen Sprache. Berlin/New York, Walter de Gruyter: 3-13.

Franck, Dorothea (1980): Grammatik und Interaktion. Stilistische Pragmatik des Dialogs und die Bedeutung deutscher Modalpartikeln. Königstein/Taunus: Scriptor.

Franco, Antonio (1989): "Modalpartikeln im Portugiesischen - Kontrastive Syntax, Semantik und Pragmatik der portugiesischen Modalpartikeln". En: Weydt, Harald (ed.): Sprechen mit Partikeln. Berlin, Walter de Gruyter: 240-255.

Giráldez, Olivia (2002): Valor ilocutivo de las partículas modales alemanas y su aplicación a la didáctica del alemán como lengua extranjera. Una propuesta metodológica. Universidad de Sevilla. Tesis Doctoral. http://fondosdigitales.us.es/tesis/tesis/361/valorilocutivo-de-las-particulas-modales-alemanas-y-su-aplicacion-a-la-didactica-del-alemancomo-lengua-extranjera-una-propuesta-metodologica/, consultado 20 de marzo, 2014.

Gornik-Gerhardt, Hildegard(1981): Zu den Funktionen der Modalpartikel 'schon' und einiger ihrer Substituentia. Tübingen: Narr. (= Tübinger Beiträge zur Linguistik 155).

Graefen, Gabriele. (2000): "Ein Beitrag zur Partikelanalyse - Beispiel: doch". Linguistik Online 6. http://www.linguistik-online.de/2_00/graefen.html, consultado 20 de marzo, 2014.

Harden, Theo (1989): "Na ja - ein vielseitiger Kommentar und seine portugiesischen Entsprechungen". En: Weydt, Harald (ed.): Sprechen mit Partikeln. Berlin, Walter de Gruyter:141-149.

Hartmann, Dietrich (1979): "Syntaktische Eigenschaften und syntaktische Funktionen der Partikeln eben, eigentlich, einfach, nämlich, ruhig, vielleicht und wohl. Zur Grundlegung 
einer diachronischen Untersuchung von Satzpartikeln im Deutschen". En: Weydt, Harald (ed.): Die Partikeln der deutschen Sprache. Heidelberg, Julius Groos: 121-138.

Heggelund, Kjell T. (2001): "Zur Bedeutung der deutschen Modalpartikeln in Gesprächen unter besonderer Berücksichtigung der Sprechakttheorie und der Daf-Perspektive". Linguistik Online 9. http://www.linguistik-online.de/9_01/Heggelund.html, consultado 20 de marzo, 2014.

Heinrichs, Werner (1981): Die Modalpartikeln im Deutschen und Schwedischen. Eine kontrastive Analyse. Tübingen: Niemeyer. (=Linguistische Arbeiten 101).

Helbig, Gerhard (1977): "Partikeln als illokutive Indikatoren im Dialog". Deutsch als Fremdsprache 14/1: 30-44.

Helbig, Gerhard (1994): Lexikon deutscher Partikeln. Berlin: Langenscheidt.

Helbig, Gerhard/Buscha, Joachim $\left({ }^{15} 1993\right)$ : Deutsche Grammatik. Ein Handbuch für den Ausländerunterricht. Leipzig/ Berlin/München: Langenscheidt.

Helbig, Gerhard/Kötz, Werner (1981): Die Partikeln. Leipzig: Verlag Enzyklopädie. Referencia en Bastert 1985.

Hentschel, Elke (1986): Funktion und Geschichte deutscher Partikeln. Ja, doch, halt und eben. Tübingen: Niemeyer. (= Germanistische Linguistik 63).

Hentschel, Elke/Weydt, Harald (1989): "Wortartenprobleme bei Partikeln". En: Weydt, Harald (ed.): Sprechen mit Partikeln. Berlin, Walter de Gruyter: 3-18.

Hentschel, Elke/Weydt, Harald $\left({ }^{4} 2013\right)$ : Handbuch der deutschen Grammatik. Berlin/New York: Walter de Gruyter.

Ikoma, Miki (2007): Prosodische Eigenschaften der deutschen Modalpartikeln. Hamburg: Dr. Kovač. (= PHILOLOGIA - Sprachwissenschaftliche Forschungsergebnisse 103).

Iwasaki, Eijiro (1972): "Abtönungspartikeln im Deutschen und Japanischen". Energeia 1: 103-110. Referencia en Malige-Klappenbach 1981.

Iwasaki, Eijiro (1977): "'Wie hieß er noch?'. Zur 'Bedeutung' von noch als Abtönungspartikel". En: Weydt, Harald (ed.): Aspekte der Modalpartikeln. Studien zur deutschen Abtönung. Tübingen, Niemeyer: 63-72.

Kastny, Andrzej (1989): "Wohl als Partikel und Modalwort und seine Übersetzungsmöglichkeiten ins Polnische". En: Weydt, Harald (ed.): Sprechen mit Partikeln. Berlin, Walter de Gruyter:196-203.

Kawashima, Atsuo (1989): "Textsorte und Partikeln im Japanischen und Deutschen". En: Weydt, Harald (ed.): Sprechen mit Partikeln. Berlin, Walter de Gruyter: 276-281.

Korošec, Vikica (1999): Der Gebrauch von Abtönungspartikeln bei den slowenischen Schülern. Pedagošca Fakulteta Maribor. http://splet-stari.fnm.unimb.si/pedagoska/programi/nem/Germanistik_files/virtual/petric/pdf/partikeln/diploma\%20 Vikica\%20Korosec\%20tretji\%20pregled.pdf, consultado 20 de marzo, 2014.

Kosaka, Koichi (1989): "Abtönungspartikel denn und Satznominalisierung". En: Weydt, Harald (ed.): Sprechen mit Partikeln. Berlin, Walter de Gruyter: 160-170.

Kriwonossow, Alexej (1977a): Die modalen Partikeln in der deutschen Gegenwartsprache. Göppingen: Kümmerle. Tesis Doctoral publicada. (= Göppinger Arbeiten zur Germanistik 214).

Kriwonossow, Alexej (1977b): "Deutsche Modalpartikeln im System der unflektierten Wortklasse". En: Weydt, Harald (ed.): Aspekte der Modalpartikeln. Studien zur deutschen Abtönung. Tübingen, Niemeyer: 176-216.

Kriwonossow, Alexej (1989): "Zum Problem der Klassifizierung der deutschen Partikeln". En: Weydt, Harald (ed.): Sprechen mit Partikeln. Berlin, Walter de Gruyter: 30-38.

Kwon, Min-Jae (2005): Modalpartikeln und Satzmodus. Untersuchungen zur Syntax, Semantik und Pragmatik der deutschen Modalpartikeln. Ludwig-Maximilians-Universität 
München.

Tesis

Doctoral.

http://edoc.ub.unimuenchen.de/archive/00004877/01/Kwon_Min-Jae.pdf, consultado 20 de marzo, 2014.

Kunow, Ilonka (1997): Diskurspartikeln im Portugiesischen. Gesprächsanalytische Studien zur Abtönung und Redeorganisation in informeller und institutioneller Kommunikation. Universität Freiburg. Tesis Doctoral. http://www.freidok.uni-freiburg.de/volltexte/272/pdf/ EP_Diss_Kunow.pdf, consultado 20 de marzo, 2014.

Lemnitzer, Lothar (2001): "Wann kommt er denn nun wohl endlich zur Sache? Modalpartikel-Kombinationen. Eine korpusbasierte Untersuchung". En: Lehr, Andrea et al. (eds.): Sprache im Alltag. Beiträge zu neuen Perspektiven in der Linguistik. Berlin, de Gruyter: 349-372.

Ličen, Marina (1989): "Die serbokroatische Partikel 'PA' und ihre deutschen Entsprechungen". En: Weydt, Harald (ed.): Sprechen mit Partikeln. Berlin, Walter de Gruyter: 171-184.

Liefländer-Koistinen, Luise (1989): "Zum deutschen doch und finnischen -han. Beobachtungen zur Übersetzbarkeit der deutschen Abtönungspartikel". En: Weydt, Harald (ed.): Sprechen mit Partikeln. Berlin, Walter de Gruyter: 185-195.

Lindqvist, Axel (1961): Satzwörter. Eine vergleichende syntaktische Studie. Göteborg: Acta Universitatis Gothoburgensis.

Lütten, Jutta (1977): Untersuchungen zur Leistung der Partikeln in der gesprochenen deutschen Sprache. Göppingen: Kümmerle. (= Göppinger Arbeiten zur Germanistik 218).

Lütten, Jutta (1979): "Die Rolle der Partikeln 'doch', 'eben', und 'ja' als KonsensusKonstitutiva in gesprochener Sprache". En: Weydt, Harald (ed.): Die Partikeln der deutschen Sprache. Berlin/New York, de Gruyter: 30-38.

Lutzeier, Peter R. (1985): Linguistische Semantik. Stuttgart: Metzler.

Malige-Klappenbach, Helene (1981): "Die Darstellung der emotionalexpressiven Partikeln im 'Wörterbuch der deutschen Gegenwartssprache'". En: Weydt, Harald (ed.): Partikeln und Deutschunterricht. Abtönungspartikeln für Lerner des Deutschen. Heidelberg, Julius Groos: 323-331.

Meibauer, Jörg (1994): Modaler Kontrast und konzeptuelle Verschiebung. Studien zur Syntax und Semantik deutscher Modalpartikeln. Tübingen: Niemeyer.

Möllering, Martina (2001): "Teaching german modal particles: a corpus-based approach". Language Learning \& Technology 5/3: 130-151. http://llt.msu.edu/vol5num3 /mollering/default.html, consultado 20 de marzo, 2014.

Moroni, Manuela C. (2010): Modalpartikeln zwischen Syntax, Prosodie und Informationsstruktur. Frankfurt a. M.: Peter Lang.

Nehls, Dietrich (1989): "German modal particles rendered by english auxiliary verbs". En: Weydt, Harald (ed.): Sprechen mit Partikeln. Berlin, Walter de Gruyter: 282-292.

Nekula, Marek (1996): System der Partikeln im Deutschen und Tschechischen: unter besonderer Berücksichtigung der Abtönungspartikeln. Tübingen: Niemeyer. (= Linguistische Arbeiten 355).

Nunes, Elaine C. R. (2012): As partículas modais da língua alemã. Um estudo com base nos contos Nachts schlafen die Ratten doch de Borchert e Berlin Bolero de Schulze. Brasil: Universidade Federal de Santa Catarina. Memoria de Máster. http://repositorio.ufsc.br/xmlui/handle/123456789/91055, consultado 20 de marzo, 2014.

O'Sullivan, Emer/Rösler, Dietmar (1989): "Wie kommen Abtönungspartikeln in deutsche Übersetzungen von Texten, deren Ausgangssprachen für diese keine direkten Äquivalente haben?" En: Weydt, Harald (ed.): Sprechen mit Partikeln. Berlin, Walter de Gruyter: 204-216. 
Poyatos, Fernando (2004): "Nuevas perspectivas lingüísticas en comunicación no verbal". En: Payrató, Lluis/Alturo, Núria/Payà, Marta (eds.): Les fronteres del llenguatge. Lingüística i comunicació no verbal. Barcelona, PPU: 57-91.

Rall, Marlene (1981): "¿Se puede enseñar la necesidad de emplear partículas intencionales? Ein Experiment mit mexikanischen Studenten". En: Weydt, Harald (ed.): Partikeln und Deutschunterricht. Abtönungspartikeln für Lerner des Deutschen. Heidelberg, Julius Groos: 123-136.

Rath, Rainer (1975): "Doch - Eine Studie zur Syntax und zur kommunikativen Funktion einer Partikel". Deutsche Sprache 3: 222-242.

Reiners, Ludwig (1961): Stilkunst. Ein Lehrbuch deutscher Prosa. München: Beck.

Ruipérez, Germán (1992): Gramática Alemana. Madrid: Cátedra.

Schiffrin, Deborah (1987): Discourse markers. Cambridge: Cambridge University Press. (= Studies in interactional sociolinguistics 5).

Schlicker, Ina (2003): Sekundäre Oralität als Form moderner Medienkommunikation. Linguistische Untersuchungen zur Sprache der Fernsehnachrichten. Ruhr-Universität Bochum. Tesis Doctoral. http://webdoc.sub.gwdg.de/ebook/dissts/Bochum/Schlicker 2003.pdf, consultado 20 de marzo, 2014.

Schmidt-Radefeldt, Jürgen (1989): "Partikeln und Interaktion im deutschportugiesischen Sprachvergleich". En: Weydt, Harald (ed.): Sprechen mit Partikeln. Berlin, Walter de Gruyter: 256-266.

Schwanzer, Viliam (1965): "Sprachelemente in kommunikativer Funktion". Philologica 17: 19-37. Referencia en Lütten 1977: 392-393.

Sekiguchi, Tsugio (1977): "Was heißt doch?". En: Weydt, Harald (ed.): Aspekte der Modalpartikeln. Studien zur deutschen Abtönung. Tübingen, Niemeyer: 19-37.

Stănescu, Speranta (1989): "Zum Status der Partikeln im Deutschen und im Rumänischen". En: Weydt, Harald (ed.): Sprechen mit Partikeln. Berlin, Walter de Gruyter: 267-275.

Stenström, Anna B. (1989): "Discourse Signals. Towards a model of analysis". En: Weydt, Harald (ed.): Sprechen mit Partikeln. Berlin, Walter de Gruyter: 561-574.

Steinmüller, Ulrich (1981): "Akzeptabilität und Verständlichkeit. Zum Partikelgebrauch von Ausländern". En: Weydt, Harald (ed.): Partikeln und Deutschunterricht. Abtönungspartikeln für Lerner des Deutschen. Heidelberg, Julius Groos: 137-148.

Thiel, Rudolf (1962): "Würzwörter". Sprachpflege 4: 71-73. Referencia en Beerbom 1991.

Thun, Harald (1984): Dialoggestaltung im Deutschen und Rumänischen: eine strukturellkontrastive Studie zu den Existimatoren. Tübingen: Narr.

Thurmair, Maria (1989): Modalpartikeln und ihre Kombinationen. Tübingen: Niemeyer. (= Linguistische Arbeiten 223).

Torregrosa, José (2011): Análisis multisistémico de las partículas modales del alemán. Departamento de Didáctica de la Lengua y la Literatura. Universidad de Barcelona. Tesis Doctoral. http://hdl.handle.net/10803/32037, consultado 20 de marzo, 2014.

Ulrich, Miorita (1989): "Personalpronomina als Abtönungspartikeln?". En: Weydt, Harald (ed.): Sprechen mit Partikeln. Berlin, Walter de Gruyter: 39-46.

Von der Gabelentz, Georg (1969): Die Sprachwissenschaft, ihre Aufgaben, Methoden und bisherigen Ergebnisse. Tübingen: Narr. Referencia en Vural 2000.

Vural, Sergül (2000): Der Partikelgebrauch im heutigen Deutsch und im heutigen Türkisch. Eine kontrastive Untersuchung. Universität Mannheim. Tesis Doctoral. http://www.unimannheim.de/mateo/verlag/diss/vural/vural.pdf, consultado 20 de marzo, 2014.

Wegener, Heide (1989): "Eine Modalpartikel besonderer Art: Der Dativus Ethicus". En: Weydt, Harald (ed.): Sprechen mit Partikeln. Berlin, Walter de Gruyter: 56-73. 
Werner, Angelika (1998): Deutsche Modalpartikeln im Kontrast zum Japanischen. Universität Siegen. Tesis Doctoral. http://www.ub.unisiegen.de/pub/diss/fb3/1999/werner/werner.pdf, consultado 20 de marzo, 2014.

Westheide, Henning (1989): "Zur ethnographischen Beschreibung von Partikelfunktionen am Beispiel Niederländisch-Deutsch". En: Weydt, Harald (ed.): Sprechen mit Partikeln. Berlin, Walter de Gruyter: 293-302.

Weydt, Harald (1969): Abtönungspartikel. Die deutschen Modalwörter und ihre französischen Entsprechungen. Bad Homburg: Gehlen.

Weydt, Harald (ed.) (1977): Aspekte der Modalpartikeln. Studien zur deutschen Abtönung. Tübingen: Niemeyer. (= Konzepte der Sprach- und Literaturwissenschaft 23).

Weydt, Harald (1981): "Methoden und Fragestellungen der Partikelforschung". En: Weydt, Harald (ed.): Partikeln und Deutschunterricht. Abtönungspartikeln für Lerner des Deutschen. Heidelberg, Julius Groos: 45-64.

Weydt, Harald (ed.) (1989): Sprechen mit Partikeln. Berlin: Walter de Gruyter.

Weydt, Harald (2001): "Partikelforschung/Particules et modalité". En: Holtus, Günter/Metzeltin, Michael/Schmitt, Christian (eds.): Lexikon der Romanistischen Linguistik. Vol. I/1. Berlin/New York, de Gruyter: 782-801.

Zifonun, Gisela/Hoffmann, Ludger/Strecker, Bruno (1997): Grammatik der deutschen Sprache.Vol. 1. Berlin/New York: de Gruyter. 